\title{
Gli accessi vascolari per emodialisi: quale futuro
}

\author{
Franco Tesio, Giacomo Panarello
}

\author{
U.O. di Nefrologia e Dialisi \\ Azienda Ospedaliera "S. Maria Degli Angeli", Pordenone
}

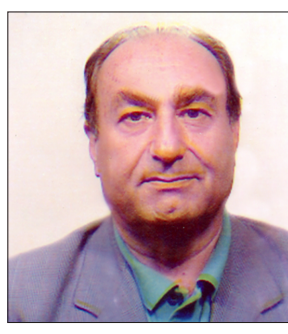

Franco Tesio

\section{T? \\ evoluzione \\ delle tecniche} emodialitiche ha permesso di allargare l'indicazione al trattamento sostitutivo dell'uremia a un sempre maggior numero di pazienti.

Ciò ha portato a una variazione demografica della popolazione degli emodializzati che ora comprende tutte le epoche della vita dalla prima infanzia fino ai grandi anziani (1-3). Inoltre il prolungamento della sopravvivenza in dialisi ha fatto spostare l'età media dei trattati sempre più in alto e determina la coesistenza oltre alla patologia di base di altre complicanze correlate al trattamento e all'accesso vascolare (4). La sopravvivenza in dialisi ormai frequentemente superiore al decennio risulta talora riconoscere come fattore critico la disponibilità di un adeguato accesso vascolare. Inoltre l'applicazione di altre metodiche terapeutiche invasive quali i trapianti, la cardiochirurgia, la chirurgia dell' aorta e l'ossigenazione extracorporea ha prodotto un'altra classe di malati che può necessitare a volte temporaneamente, ma talora permanentemente il trattamento dialitico. Quindi l'allestimento e il mantenimento di un affidabile accesso vascolare per emodialisi rappresenta ancor oggi una sfida aperta per $\mathrm{i}$ nefrologi ed i chirurghi vascolari. Attualmente l'incannulamento tramite uno o più cateteri di una vena centrale rappresenta il più rapido e facile accesso, idoneo a garantire un adeguato flusso ematico per qualsivoglia trattamento emodialitico. La fistola arteriovenosa interna di Cimino-Brescia è invece l'accesso più sicuro e certamente meno lesivo disponibile.

\section{Accessi vascolari centrali}

Questo accesso che permette di effettuare il trattamento dialitico nell'immediato postoperatorio, non è comunque scevro di rischi; la diffusione della metodica porta, sempre più frequentemente e per necessità, a trascurare le conseguenze che ne possono derivare. Schwab S. (5) presentando un corso sull' argomento doveva ammettere: " $l i$ odio ma non posso farne a meno".
Gl'incidenti acuti, non rari, legati all'inserimento, sono le aritmie atriali e meno frequentemente, quelle ventricolari, la puntura dell'arteria, il pneumotorace, l'emotorace, la perforazione dei vasi, l'embolia. Gl'incidenti tardivi sono le infezioni, le trombosi e le stenosi delle vene centrali ospitanti il catetere (6).

Queste complicazioni ci devono far riflettere su come l'inserimento di un corpo estraneo, che viene allocato per svariati centimetri in un vaso centrale, non sia scevro di rischi e possa compromettere la possibilità futura di ulteriori accessi. Per quanto nella nostra esperienza, l'uso di due cateteri separati di materiale biocompatibile, posizionati e gestiti adeguatamente, abbia provocato una svolta nell'uso degli accessi venosi centrali (7), non riteniamo che possa comunque annullare questi rischi.

\section{Fistole arterovenose interne}

Il "golden standard" degli accessi vascolari per emodialisi non è anch'esso scevro di complicazioni, specie se valutato in considerazione delle caratte- 


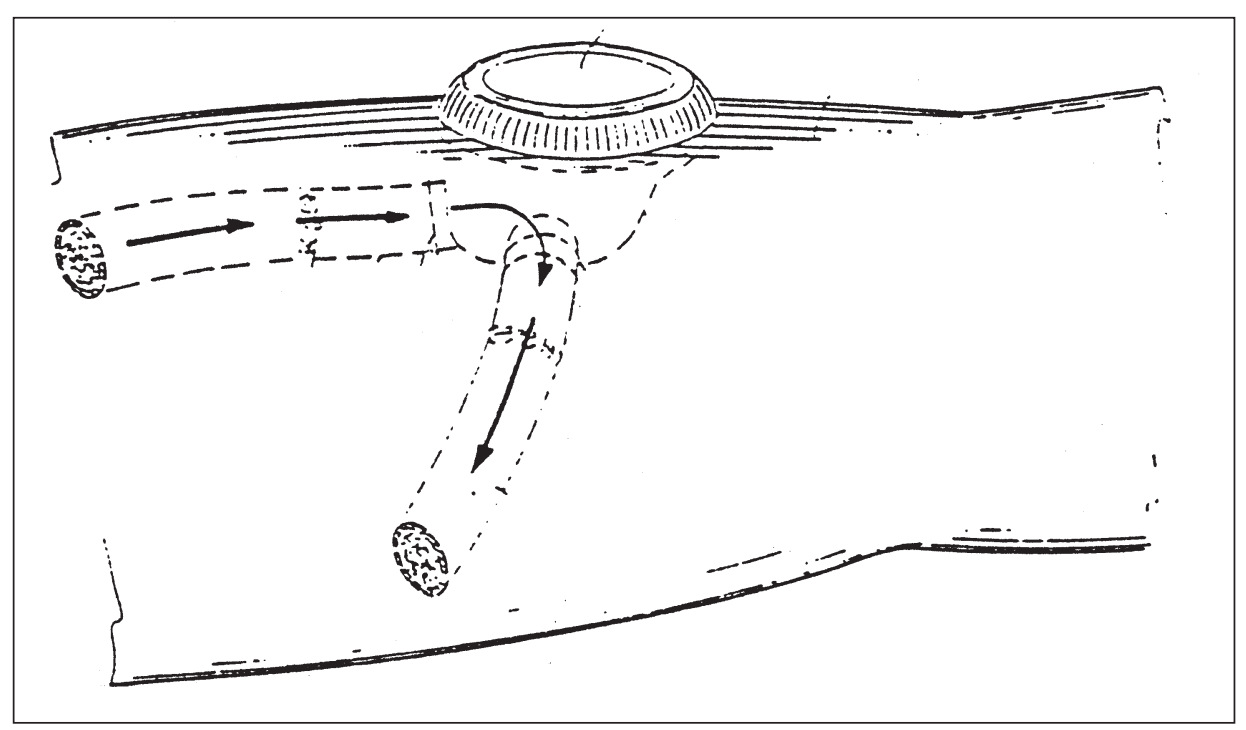

Fig. 1

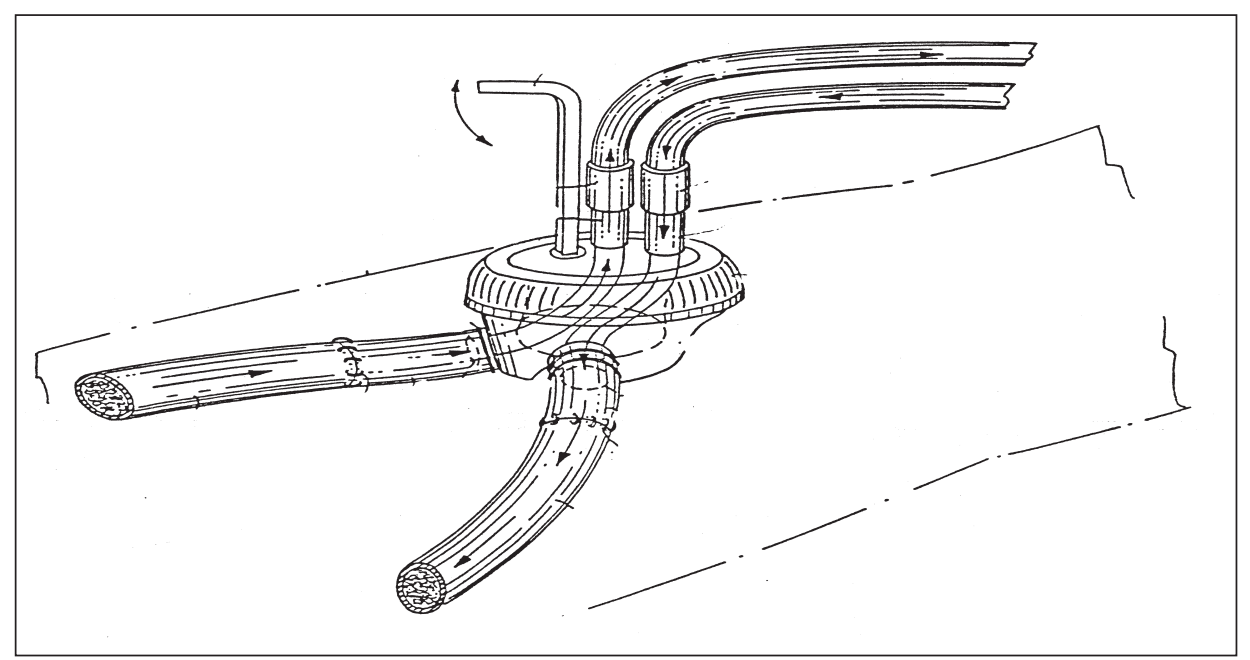

Fig. 2

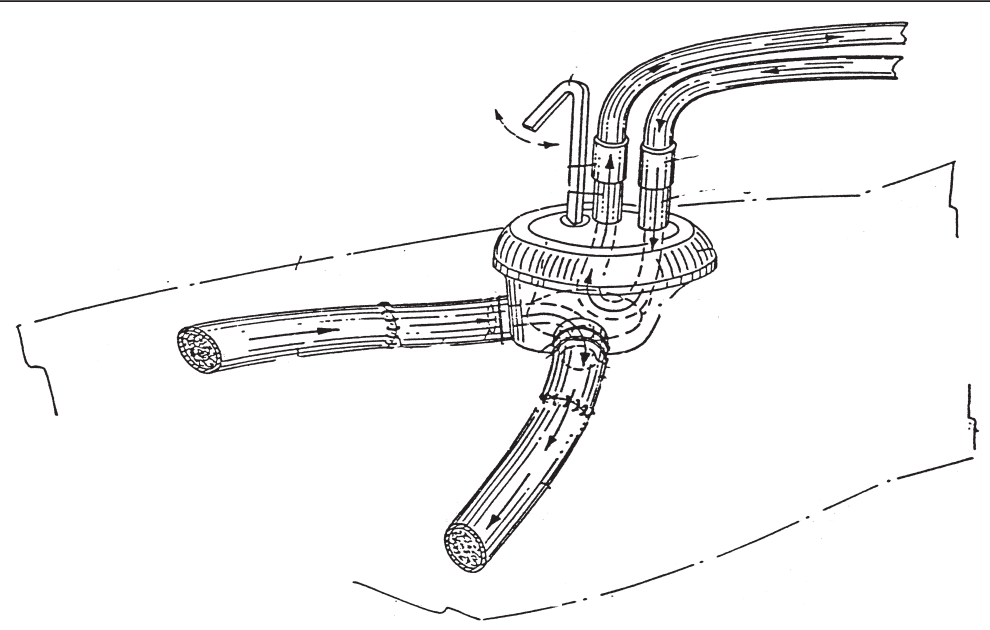

Fig. 3 ristiche di maggioranza dei soggetti che accedono all'emodialisi. Inoltre non sempre è possibile allestirlo o mantenerlo negli anni (8). Il letto vascolare, infatti, è sovente già compromesso prima dell'inizio della dialisi e la puntura ripetuta della fistola esaurisce rapidamente le possibilità d'uso. La fistola richiede inoltre un periodo più $\mathrm{o}$ meno lungo di "maturazione" prima di essere utilizzata e quindi presuppone nell'urgenza il ricorso al catetere venoso centrale. Da non trascurare poi le variazioni all'emodinamica locale e centrale che una fistola arterovenosa causa nei soggetti in fase di labile compenso cardiaco.

\section{Nuovo accesso vascolare}

Come detto nell'introduzione, l'accesso ai vasi rappresenta ancor oggi il "tallone d'Achille" del trattamento emodialitico.

L'accesso ideale dovrebbe permettere l'immediato utilizzo, e annullare, o quanto meno ridurre, le complicazioni derivanti dalle metodiche attuali, senza compromettere il letto vascolare, per lasciare sempre la possibilità di un ulteriore intervento.

Gli accessi vascolari tipo "bottone", sono comparsi negli USA nel 1980 (Biocarbon Hemasite) $(9,10)$, ma il loro uso si ridusse molto rapidamente perché provocavano facilmente infezioni non solo locali ma anche sistemiche. Una proposta francese, il Bioclip (11) non ebbe miglior esito. Una nostra breve esperienza con il Biocarbon ci ha comunque confermato l'importanza che questo tipo di accesso vascolare, senza puntura venosa, poteva avere nella vita del dializzato. Era però necessario disporre di un sistema che riducesse le infezioni, in prevalenza favorite dai microtraumi, che le manovre di manipolazione dell'accesso, attacco e stacco dalle linee dialitiche, causavano. Era altresì indispensabile disporre di un presidio che permettesse una facile disostruzione del sistema in caso di trombosi, senza necessitare la sua sostituzione. Il "bottone" doveva inoltre essere di dimensioni contenute ed avere uno spessore non superiore al centimetro 
per poter essere applicato senza problemi, anche distalmente, in persone di piccola corporatura. Presentiamo la nostra proposta ritenendo che il futuro dell' accesso vascolare, non solo per emodialisi, debba seguire questa via.

Il "bottone" da noi ideato (12) è costituito da un corpo centrale collegato tramite due segmenti vascolari artificiali (PTFE o tessuti simili) a una arteria e una vena (Fig. 1) In pratica corrisponde a quanto si prepara quando occorre creare un ponte tra due vasi nativi distanti tra loro. Il corpo è costituito da una valvola rotante "rubinetto" con due posizioni che permettono due passaggi indipendenti (Fig. 2). In condizione di riposo si ottiene il passaggio diretto del sangue arterioso nella vena ricevente mentre il corpo del bottone risulta da un cappuccio avente nel suo interno una spugna imbevuta di disinfettante.

All'inizio della dialisi si rimuove il cappuccio, si disinfetta, si lava con soluzione fisiologica il condotto esterno e tramite la brugola (Fig. 3) si collega prima l'arteria e poi la vena con un movimento orario. Si viene così a creare una doppia via separata fra il lato arterioso e quello venoso. La stessa manovra viene ripetuta al termine della seduta, continuando sempre in senso orario, chiudendo prima il lato arterioso e poi quello venoso (Fig. 3). Il percorso a U esterno, viene saturato con soluzione disinfettante e poi ricoperto del cappuccio esterno. Il bottone ha le dimensioni esterne di $1.5 \mathrm{~cm}$ e nel punto di emergenza presenta un colletto che permette alla cute di aderire strettamente al corpo del sistema. La parte interna profonda $11 \mathrm{~mm}$, per prevenire le lesioni microtraumatiche favorenti processi settici, è rivestita da una camicia in dacron; fra di essa e il corpo del bottone rigido, vi è posto un cuscino di 1-2 mm di silicone liquido con funzione di ammortizzatore. Il corpo centrale è facilmente svitabile per permettere un rapido accesso ai vasi, nel caso in cui si rendessero necessarie eventuali manovre di disostruzione.

\section{Conclusione}

È nostra opinione che la soluzione avanzata possa rappresentare una svolta nella realizzazione di un accesso vascolare a lunga permanenza, che eviti la puntura ripetuta del letto venoso, che risparmi i vasi a monte e quindi permetta la reiterata ripetizione dell'accesso. Il silicone liquido usato come ammortizzatore dovrebbe ridurre il rischio infettivo che ha determinato l'insuccesso clinico in precedenti esperienze.

segreteria.dialisi@aopn.fvg.it

\section{BIBLIOGRAFIA}

1. Sheth RD, Kale AS, Brewer ED, Brandt ML, Nuchtern JG, Goldestein AB. Successful use of Tesio catheteres in pediatric patients receiving hemodialysis. Am J Kidney Dis 2001; 38 (3): 553-9.

2. Di Benedetto A, Buono A, Cappabianca F, Merinelli G. Dialysais in elderly patients. Lancet 2001; 358: 1963 .

3. Di Benedetto A, Barone V, Nuzzi F, Sorice M, Rocino A, Sorice P. Il cateterismo della vena giugulare interna secondo Tesio. Atti X Convegno Reg SIN, Sezione Campania, 1995; 26: 119-22.

4. Chazan JA, London MR, Pono Ml. Long term survival of vascular access in a large chronic hemodialysis population. Nephron 1995; 69: 228-33.

5. Schwab SJ, Beathard G. The hemodialysis catheter conundrum: hate living with them, but can't live without them. Kidney Int 1999; 56: 1-17.
6. Pang YF. Acute vascular access: new advances. Advances in renal replacement therapy 1994; 1 (2): 90-98.

7. Baracetti S. Indication for the use of central venous catheters as vascular access for hemodialysis. The Journal of Vascular Access 2001;2: 20-7.

8. Albers FJ. Causes of hemodialysis access failure. Advances in renal replacement therapy 1994; 1 (2): 107-18

9. Golding AL, Nissenson AR, Higgins R, Raible D. Carbon transcutaneous access device (CTAD). Trans Am Soc Artif Intern Organs 1980; 26: 105-10.

10. Saphiro FL, Keshaviah PR, Carlson LD, et al. Blood access without percutaneous punctures. Proc Dialysis Transplant Forum 1980; 10: 130-7.

11. Bonnaud P, Jehenne G, KoaMan N. Le carboclip, un nouvel access vasculaire atraumatique pour hemodialyse. Nephrologie 1994; 15 (2): 181-4.

12. Tesio F. Vascular access device, in particular for purification treatments of the blood. USA Patent 1990; 4, 898, 669 . 